\title{
Internamentos por septicemia nas capitais brasileiras, no período de 1999 a 2016 e a sua relação com o Índice de Desenvolvimento Humano
}

Hospitalizations for septicemia in Brazilian capitals, from 1999 to 2016 and their relationship with

the Human Development Index

Hospitalizaciones por septicemia en capitales brasileñas, de 1999 a 2016

Y su relación con el Índice de Desarrollo Humano

Recebido: 12/04/2021 | Revisado: 17/04/2021 | Aceito: 23/04/2021 | Publicado: 08/05/2021

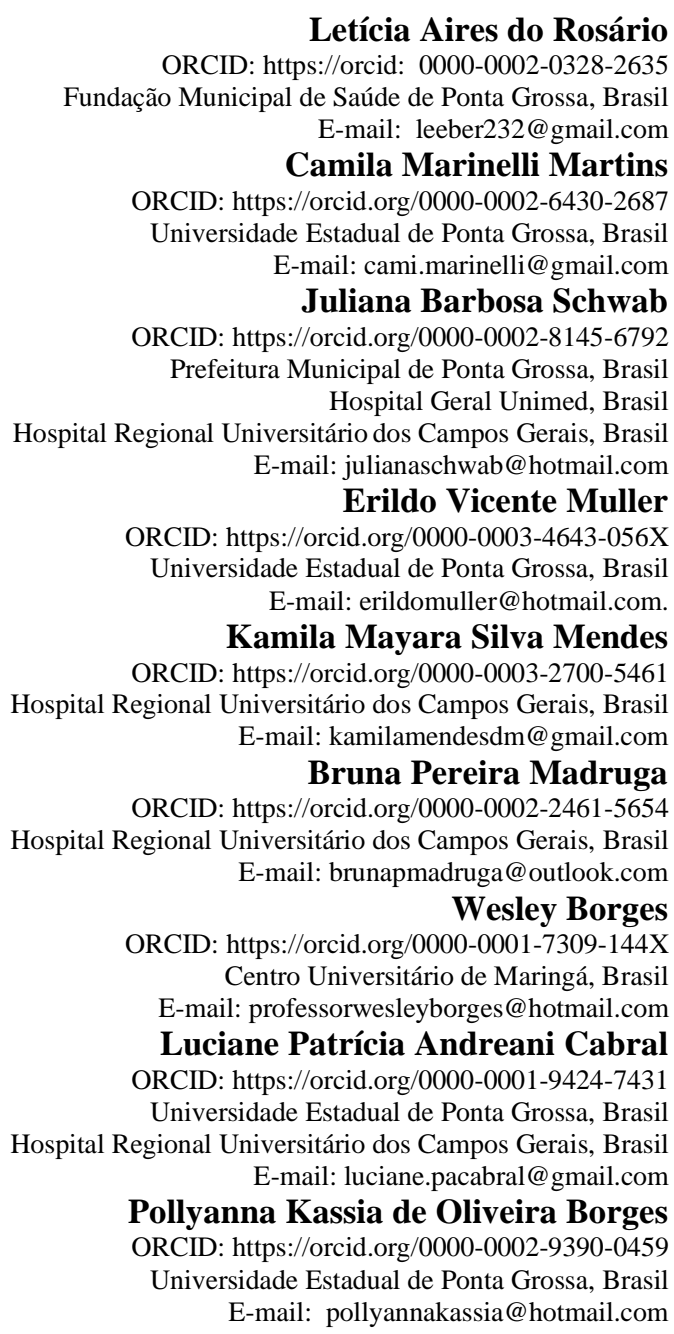

\section{Resumo}

Objetivos: demonstrar a série histórica de internamentos por sepse no Brasil, quais as capitais e regiões mais acometidas, bem como, se essas internações tiveram crescimento, em que proporção ocorreram e qual a relação com o Índice de Desenvolvimento Humano (IDH). Métodos: Foi realizado um estudo epidemiológico de série temporal com dados dos internamentos por septicemia nas capitais brasileiras de 1999 a 2016, obtidos do Sistema de Informações Hospitalares do Ministério da Saúde (SIH) e IDH das mesmas no período. Foram calculados os indicadores de internamentos para a metade de cada triênio do período obtido. Modelos de regressão linear e de Poisson foram realizados para verificar a tendência temporal e coeficientes de correlação de Pearson ou Spearman para correlação com o IDH. Resultados: houve aumento significativo de internamentos em algumas capitais, tais como: Boa Vista, Maceió, Porto Velho e Rio de Janeiro, e decréscimo em Campo Grande e Goiânia. A maior média de internamentos ocorreu em Porto Velho com 26.443,7 e a menor média em Teresina 171,3. Os coeficientes de correlação entre o total de internamentos e o IDH de cada capital registraram correlação positiva e significativa para Belo Horizonte, Boa Vista, 
São Paulo e Vitória. Conclusão: os internamentos por sepse tiveram mais acréscimos que decréscimos e a correlação com o IDH mostrou que quanto maior o IDH, maior fica o índice de internamentos, sugerindo que com taxas maiores desse IDH, torna-se possível fornecer uma estrutura adequada e um melhor desempenho no reconhecimento e desfecho da sepse.

Palavras-chave: Pesquisa em sistemas de saúde oública; Índice de desenvolvimento humano, IDH; Saúde pública; Epidemiologia; Sepse.

\begin{abstract}
Objectives: to demonstrate the historical series of hospitalizations for sepsis in Brazil, which capitals and regions are most affected, as well as whether these hospitalizations grew and in what proportion they occurred and what is the relationship with the Human Development Index (HDI). Methods: An epidemiological time series study was conducted with data on hospitalizations for septicemia in Brazilian capitals from 1999 to 2016 obtained from the Hospital Information System of the Ministry of Health (SIH) and their HDI in the same period. The hospitalization indicators were calculated for half of each three-year period. Linear and Poisson regression models were performed to verify the temporal tendency and Pearson or Spearman correlation coefficients for correlation with the HDI. Results: there was a significant increase in hospitalizations in some capitals, such as: Boa Vista, Maceió, Porto Velho and Rio de Janeiro, and a decrease in Campo Grande and Goiânia. The highest average of hospitalizations occurred in Porto Velho with 26,443.7 and the lowest average in Teresina 171.3. The correlation coefficients between the total number of hospitalizations and the HDI of each capital, showed a positive and significant increase for Belo Horizonte, Boa Vista, São Paulo and Vitória. Conclusion: hospitalizations for sepsis had more increases than decreases, and the correlation with the HDI showed that the higher the HDI, the greater the hospitalizations, suggesting that with higher rates, it becomes possible to provide an adequate structure and better performance in early recognition and outcome of sepsis.

Keywords: Research in public health systems; Human development index, HDI; Public health; Epidemiology; Sepsis.
\end{abstract}

\title{
Resumen
}

Objetivos: demostrar la serie histórica de hospitalizaciones por sepsis en Brasil, qué capitales y regiones son las más afectadas, así como, si estas hospitalizaciones tuvieron crecimiento, en qué proporción ocurrieron y cuál es la relación con el Índice de Desarrollo Humano (IDH). ). Métodos: se realizó un estudio epidemiológico de series de tiempo con datos de hospitalizaciones por septicemia en capitales brasileñas de 1999 a 2016, obtenidos del Sistema de Información Hospitalaria del Ministerio de Salud (SIH) y su IDH en el período. Los indicadores de hospitalización se calcularon para la mitad de cada período de tres años. Se realizaron modelos de regresión lineal y de Poisson para verificar la tendencia temporal y los coeficientes de correlación de Pearson o Spearman para la correlación con el IDH. Resultados: hubo un aumento significativo de las hospitalizaciones en algunas capitales, como: Boa Vista, Maceió, Porto Velho y Río de Janeiro, y una disminución en Campo Grande y Goiânia. El promedio más alto de hospitalizaciones ocurrió en Porto Velho con 26.443,7 y el promedio más bajo en Teresina 171,3. Los coeficientes de correlación entre el número total de hospitalizaciones y el IDH de cada capital registraron una correlación positiva y significativa para Belo Horizonte, Boa Vista, São Paulo y Vitória. Conclusión: las hospitalizaciones por sepsis tuvieron más aumentos que disminuciones y la correlación con el IDH mostró que a mayor IDH, mayor tasa de hospitalizaciones, sugiriendo que con mayores tasas de este IDH, es posible proporcionar una estructura adecuada y una mejor 1. Desempeño en el reconocimiento y resultado de la sepsis.

Palabras clave: Investigación en sistemas de salud pública; Índice de desarrollo humano, IDH; Salud pública; Epidemiología; Septicemia.

\section{Introdução}

A sepse, a partir do consenso Sepsis-3 emitido pela Society of Critical Care Medicine (SCCM) e a European Society of Intensive Care Medicine (ESICM), é definida pela presença de disfunção orgânica ameaçadora à vida e a resposta desregulada do hospedeiro a uma infecção (Singer et al., 2016).

Segundo o Instituto de Sepse Latino-Americano [ILAS] (2018), a ocorrência mundial de sepse é estimada em 17 milhões de casos anualmente, sendo que, de 2003 a 2007, houve um crescimento de 285 mil casos, somente nos Estados Unidos.

Estudos de países como Alemanha e Espanha demonstraram taxas de 17\% e 12,4\% de ocorrência de sepse, sendo a incidência estimada de 25 casos de sepse grave atendidos em UTI por 100.000 habitantes por ano na Espanha, em relação aos óbitos foram 40,4\%, 53,4\% respectivamente (Marx et al., 2016; Blanco et al., 2008). O que evidencia que mesmo países desenvolvidos ainda apresentam significativas taxas de incidência e mortalidade, sendo necessária a busca incessante de diminuição das hospitalizações e óbitos por sepse. 
No Brasil, um estudo realizado por Machado e colaboradores (2017), em UTIs em determinadas regiões brasileiras registrou uma incidência de 16\% (133 casos) em um único dia, tendo óbito como desfecho em 55\% desses casos, a proporção de leitos de UTI ocupados por sepse foi de $30 \%$, demonstrando o grande impacto sobre a assistência à saúde e a economia do país.

As pesquisas sobre sepse, ainda escassas nos países de média e baixa renda, são de interesse mundial, pois se estima que uma significativa porcentagem da incidência se dá nesses países (Fleischmann et al., 2016).

Evidências científicas e consensos internacionais têm atestado conforme se deve agir e quais os principais fatores envolvidos nos maus prognósticos relacionados a sepse, alguns dos pontos principais são a identificação precoce, que é extremamente essencial e o tratamento indicado, que deve ser iniciado em tempo oportuno (Levy et al., 2015; Kim \& Park, 2018).

O bundles recomendado para um resultado mais favorável, de acordo com o sepsis-3(Singer et al., 2016), dispõe de algumas medidas importantes, entre elas o tratamento adequado que deve ser iniciado em até uma hora, a antibioticoterapia de amplo espectro o mais brevemente possível, ainda nesse pacote de medidas recomendadas citamos a ressuscitação volêmica associada a constante reavaliação (Lam et al., 2017).

Todavia o impasse relacionado à obtenção de melhores resultados está correlacionado com a estrutura e tecnologia precária, equipe multiprofissional de saúde despreparada para o reconhecimento precoce, falta de adesão de protocolos de manejo indicados a partir de evidências científicas, seja por falta de recursos de saúde ou conhecimento inadequado, entre outros aspectos limitadores (Westphal \& Lino, 2015).

Sendo assim, esses aspectos estão de modo contínuo associados a questões socioeconômicas. Isto posto, o Índice de Desenvolvimento Humano (IDH) valida-se como um recurso efetivo de obter conhecimento sobre os mais importantes aspectos das regiões e as condições de saúde de uma determinada população (Programa das Nações Unidas para o Desenvolvimento (PNUD), Instituto de Pesquisa Econômica Aplicada (IPEA) \& Fundação João Pinheiro (FJP), 2018).

Rudd et al. (2020) demonstraram que a população neonatal e idosa são as mais acometidas. Na população idosa, há o aumento da expectativa de vida e, com o avanço da idade, ocorrem diversas mudanças fisiológicas que levam à ocorrência de doenças crônicas não transmissíveis e consequente aumento no número de hospitalizações, exposições a procedimentos complexos e invasivos, entre outros (Duarte et al., 2019; Kahlon et al., 2015).

No que se refere à população neonatal, destacamos o problema relativo às complicações associadas ao pré-natal de baixa qualidade, que podem ocasionar partos prematuros e suas possíveis consequências (Alves et al., 2018).

O objetivo deste estudo foi descrever a série histórica de internamentos por sepse no Brasil entre 1999-2016, enfatizando capitais e regiões mais acometidas e sua correlação com o Índice de Desenvolvimento Humano (IDH) das mesmas, a fim de fornecer conhecimento sobre a incidência dos casos ao decorrer da linha histórica e base para planejamento de ações pelas equipes de saúde.

\section{Metodologia}

\section{Delineamento do estudo}

Foi realizado um estudo epidemiológico do tipo ecológico quantitativo de série temporal para análise histórica dos internamentos por sepse no Brasil (Pereira et al., 2018), sendo esses internamentos ocorridos em três triênios, 1999/2000/2001, 2009/2010/2011 e 2014/2015/2016. 


\section{Coleta dos dados}

Os dados foram obtidos em uma base de acesso público do Brasil, o sistema de informação hospitalar (SIH) nos anos de 1999 a 2016, no TABNET do Departamento de Informática do SUS (DATASUS), que armazena inúmeras informações geradas pelo SUS, tais como: informações epidemiológicas e de morbidade, demográficas e socioeconômicas, financeiras, entre outras.

Os dados de internamentos por sepse, causas mal definidas e total de população foram coletados para o cálculo dos indicadores de internamentos para a metade de cada triênio. Para os anos de 2012-2016 não havia o total da população disponível, então se realizou a projeção para tais anos da seguinte forma:

1) calculou-se a média do crescimento populacional do triênio 2009-2011 em cada capital pelo quociente (população 2010/população 2009) / (população 2011/2010); 2) a média de crescimento populacional foi multiplicada pela população do ano anterior com dado disponível (2011), em cada capital; 3) no valor encontrado na multiplicação do passo 2 foi considerada a projeção populacional da capital específica. Sucessivamente as populações dos anos seguintes foram projetadas, a partir da projeção do triênio anterior.

Os dados do IDHM das capitais foram obtidos a partir de dados disponíveis no endereço eletrônico do Programa das Nações Unidas para o Desenvolvimento (PNUD). Foram considerados os índices dos anos 1991, 2000 e 2010 (PNUD, 2013).

Desta forma, os dados analisados foram: número de internamentos, população e IDH de cada capital em cada ano da série.

\section{Análise dos dados}

$\mathrm{Na}$ análise da tendência temporal, a média dos internamentos se deu pela soma de cada ano correspondente ao seu triênio, e então dividiu-se por três, os coeficientes brutos dos internamentos, das causas mal definidas e por todas as causas, foi utilizada a média dividida pela população do meio do triênio e após multiplicada por cem mil. O coeficiente de internamentos foi compensado pelas causas mal definidas para minimizar a influência desses dados mal definidos sobre os internamentos por sepse, conforme metodologia de Muller e Gimeno (2015), a qual se obtém a partir da seguinte fórmula: média dos internamentos por sepse, somados a média dos casos mal definidos, multiplicados por média dos internamentos por sepse, divididos por internamentos por todas as causas, subtraídos pela média dos casos mal definidos, sendo $\mathrm{A}^{2}$ o número compensado de internamentos pela causa específica. $A^{2}=(A+B) * A /(D-B)$. E por último o coeficiente foi padronizado pela população brasileira, isso foi realizado para minimizar o impacto das diferentes distribuições populacionais por faixa etária entre as capitais, e foi calculado da seguinte forma: coeficiente compensado pelos mal definidos divididos pela população de cada capital, multiplicado pela proporção da população brasileira, multiplicada por cem mil habitantes.

Os coeficientes foram testados para distribuição normal com o teste de Shapiro-Wilk e houve coeficientes simétricos e assimétricos. Análises estatísticas foram realizadas para verificar a tendência temporal com a análise de regressão simples de todas as capitais como variável dependente e o triênio como variável independente. Modelos de regressão linear e de Poisson foram utilizados para distribuições simétricas e não simétricas, respectivamente.

Para verificar a correlação com o IDH, calcularam-se os coeficientes de correlação e se utilizaram os coeficientes de Pearson ou Spearman para distribuições simétricas e não simétricas, respectivamente. Todas as análises foram realizadas em ambiente R com pacote "stats" (R Core Team, 2017) e consideradas significativas quando $p<0,05$.

\section{Resultados}

A Tabela 1 mostra a tendência temporal do total de internamentos no Brasil em 1999-2001, 2009-2011 e 2014-2016 de todas as capitais do país. A análise apontou aumento significativo em Boa Vista (p-valor 0,038), Maceió (p-valor <0,001), 
Porto Velho (p-valor <0,001) e Rio de Janeiro $(<0,001)$ e diminuição para Campo Grande (p-valor 0,041) e Goiânia (p-valor $<0,001$ ) (Tabela 1).

Table 1. Média, mediana e desvio padrão (DP) do total de internamentos no Brasil, regressão linear dos coeficientes e análise de tendência temporal (cada triênio) de todas as capitais.

\begin{tabular}{|c|c|c|c|c|c|c|}
\hline & \multicolumn{6}{|c|}{ Total of Internship in 1999-2001, 2009-2011 and 2014-2016 } \\
\hline & Média & Mediana & DP & Coeficiente de regressão (IC 95\%) & p-valor & $\mathbf{R}^{2}$ \\
\hline \multicolumn{7}{|l|}{ Centro-oeste } \\
\hline Cuiabá & 4218.0 & 1931.0 & 3975.9 & $3,447.5(-21,718.4-28,613.4)$ & 0.332 & 0.752 \\
\hline Campo Grande & 459.0 & 539.0 & 194.7 & $-0.067(-0.132--0.002)$ & 0.041 & 0.025 \\
\hline Goiânia & 720.3 & 797.0 & 274.2 & $-0.105(-0.157--0.0535)$ & $<0.001$ & 0.076 \\
\hline Brasília & 2624.0 & 2267.0 & 1480.1 & $1,447.5(-2.5-5,375.8)$ & 0.134 & 0.956 \\
\hline \multicolumn{7}{|l|}{ Nordeste } \\
\hline São Luís & 1109.3 & 623.0 & 1239.3 & $947.5(-9,201.8-11,096.7)$ & 0.446 & 0.585 \\
\hline Teresina & 171.3 & 165.0 & 66.7 & $38.0(-658.9-734.9)$ & 0.614 & 0.324 \\
\hline Fortaleza & 1794.3 & 1401.0 & 830.1 & $-673.5(-6,839.3-5,492.3)$ & 0.397 & 0.658 \\
\hline Salvador & 2524.0 & 2922.0 & 705.9 & $0.003(-0.023-0.031)$ & 0.786 & 0.001 \\
\hline Aracaju & 1588.7 & 1656.0 & 1242.4 & $-570.0(-14,596.3-13,456.3)$ & 0.697 & 0.210 \\
\hline Maceió & 2666.7 & 806.0 & 3304.5 & $1.371(1.331-1.411)$ & $<0.001$ & 0.738 \\
\hline Recife & 4421.3 & 5593.0 & 3251.9 & $666.0(-39,776.9-41,108.9)$ & 0.869 & 0.042 \\
\hline Natal & 6483.3 & 6634.0 & 1038.2 & $-628.0(-11,133.0-9,877.0)$ & 0.586 & 0.366 \\
\hline João Pessoa & 4698.3 & 3166.0 & 2756.0 & $-2,415.5(-19,277.1-14,446.1)$ & 0.320 & 0.768 \\
\hline \multicolumn{7}{|l|}{ Sul } \\
\hline Curitiba & 1857.0 & 2359.0 & 1223.8 & $1,144.0(-4,379.9-6,667.9)$ & 0.231 & 0.874 \\
\hline Florianópolis & 958.3 & 1040.0 & 214.5 & $162.5(-1,616.6-1,941.4)$ & 0.453 & 0.574 \\
\hline Porto Alegre & 7355.0 & 5525.0 & 4067.5 & $-3,746.0(-23,883.1-16,391.1)$ & 0.255 & 0.848 \\
\hline \multicolumn{7}{|l|}{ Sudeste } \\
\hline Rio de Janeiro & 1673.7 & 979.0 & 1204.1 & $0.669(0.631-0.706)$ & $<0.001$ & 0.750 \\
\hline São Paulo & 4303.3 & 4579.0 & 2107.1 & $2.093 .5(-939.9-5,126.9)$ & 0.072 & 0.987 \\
\hline Belo Horizonte & 5817.3 & 6194.0 & 2878.5 & $2,860.0(-1,284.8-7,004.8)$ & 0.072 & 0.987 \\
\hline Vitória & 3308.7 & 3611.0 & 2273.6 & $2,258.5(-1,068.3-5,585.3)$ & 0.073 & 0.987 \\
\hline \multicolumn{7}{|l|}{ Norte } \\
\hline Macapá & 1011.7 & 995.0 & 634.2 & $-304.5(-7,372.7-6,763.6)$ & 0.681 & 0.231 \\
\hline Manaus & 2694.3 & 2126.0 & 1984.5 & $1,387.5(-16,640.5-19,415.5)$ & 0.507 & 0.489 \\
\hline Palmas & 997.0 & 1231.0 & 542.3 & $-76.0(-6,898.4-6,746.4)$ & 0.910 & 0.020 \\
\hline Porto Velho & 26443.7 & 745.0 & 44652.6 & $3.748(3.708-3.793)$ & $<0.001$ & 0.752 \\
\hline Rio Branco & 1873.0 & 1736.0 & 543.6 & $530.5(-977.0-2,038.0)$ & 0.140 & 0.952 \\
\hline Belém & 2265.3 & 2123.0 & 1365.1 & $786.5(-13,390.2-14,963.1)$ & 0.609 & 0.576 \\
\hline Boa Vista & 178.3 & 187.0 & 127.2 & $127.0(31.6-222.3)$ & 0.038 & 0,997 \\
\hline
\end{tabular}

Fonte: Autores.

Ainda na Tabela 1, destacam-se as maiores médias de internamentos ocorridas de 1999 a 2016, sendo elas: Porto Velho (26.443,7), Porto Alegre $(7.355,0)$, Natal com $(6.483,3)$ e Belo Horizonte com $(5.817,3)$ casos. De outro modo, as 
menores médias de ocorrência ocorreram em Teresina (171,3), Boa Vista (178,3), Campo Grande (459,0) e Goiânia com $(720,3)$.

Nas Figuras 1 a 5 é descrita a distribuição temporal dos coeficientes de internação hospitalar por sepse em cada capital, separadas por regiões do país.

Figura 1. Total de internamentos nos triênios de 1999-2001, 2009-2011 e 2014-2016 na região Centro-Oeste do Brasil.

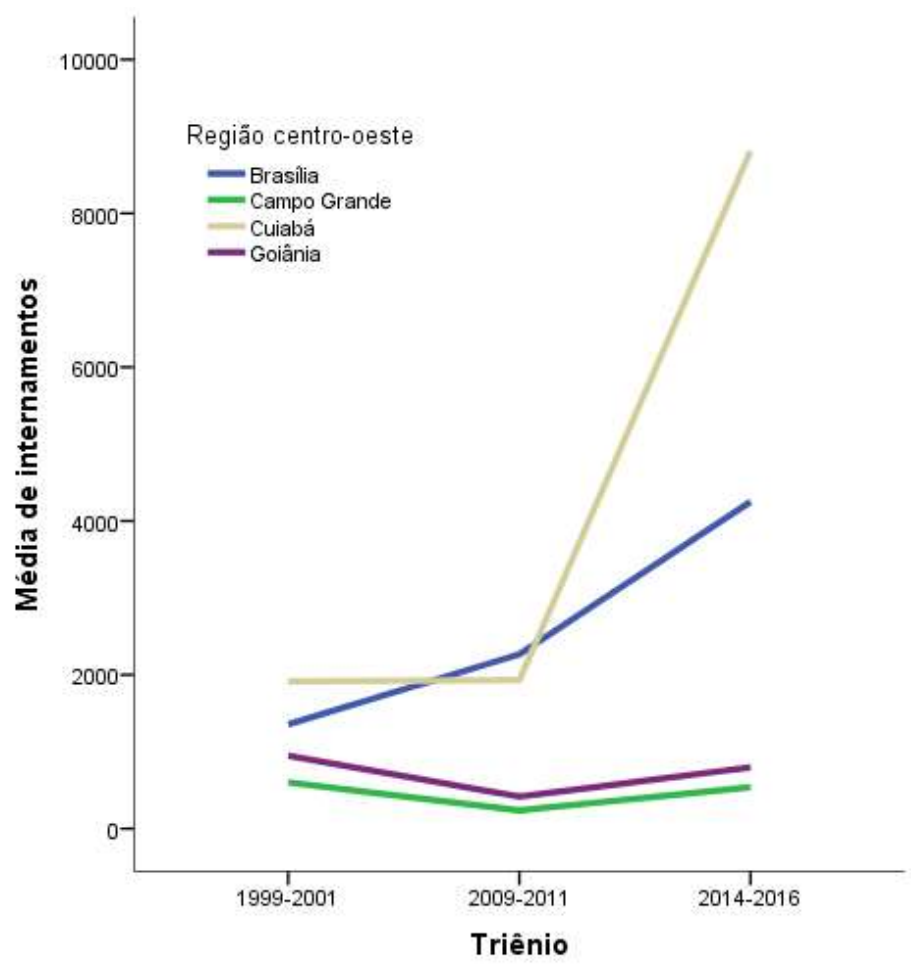

Fonte: Autores.

Figura 2. Total de internamentos nos triênios de 1999-2001, 2009-2011 e 2014-2016 na região Nordeste do Brasil.

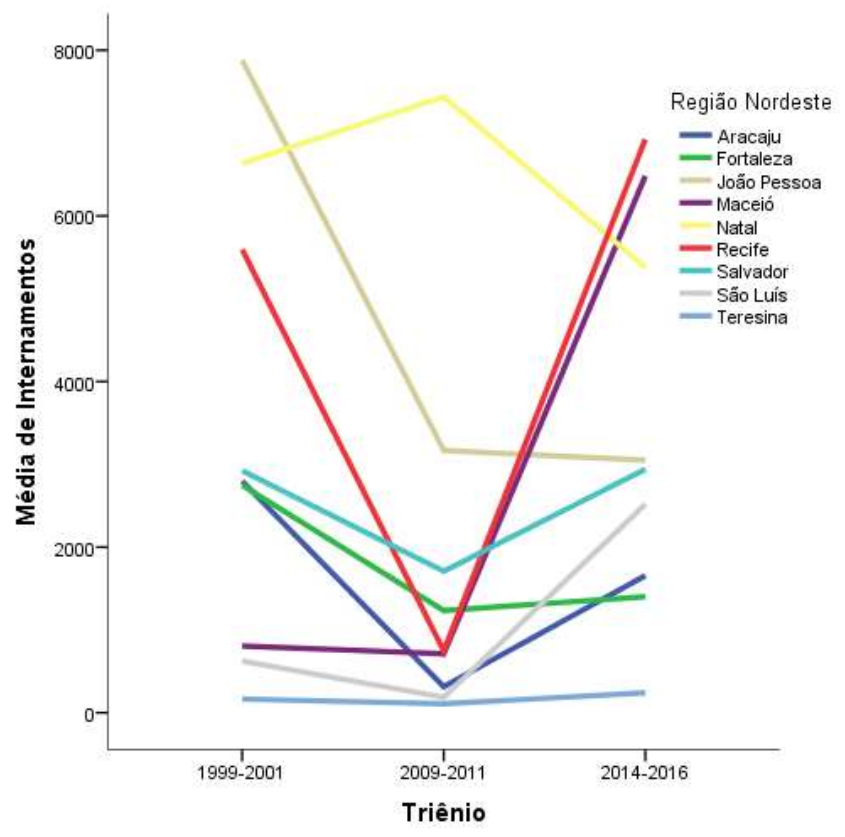

Fonte: Autores. 
Research, Society and Development, v. 10, n. 5, e31610514977, 2021

(CC BY 4.0) | ISSN 2525-3409 | DOI: http://dx.doi.org/10.33448/rsd-v10i5.14977

Figura 3. Total de internamentos nos triênios de 1999-2001, 2009-2011 e 2014-2016 na região Norte do Brasil.

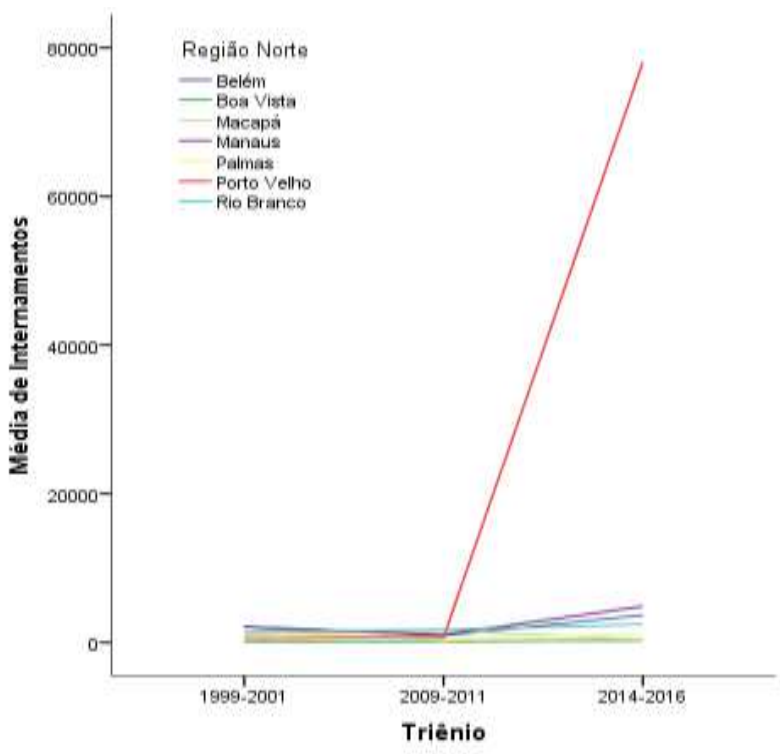

Fonte: Autores.

Figura 4. Total de internamentos nos triênios de 1999-2001, 2009-2011 e 2014-2016 na região Sudeste do Brasil.

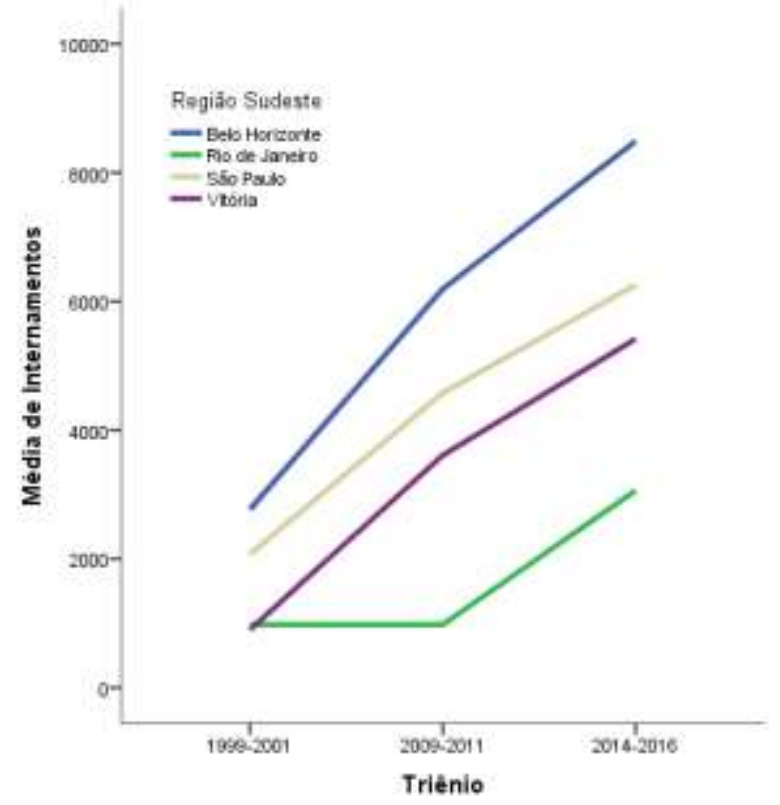

Fonte: Autores. 
Figura 5. Total de internamentos nos triênios de 1999-2001, 2009-2011 e 2014-2016 na região Sul do Brasil.

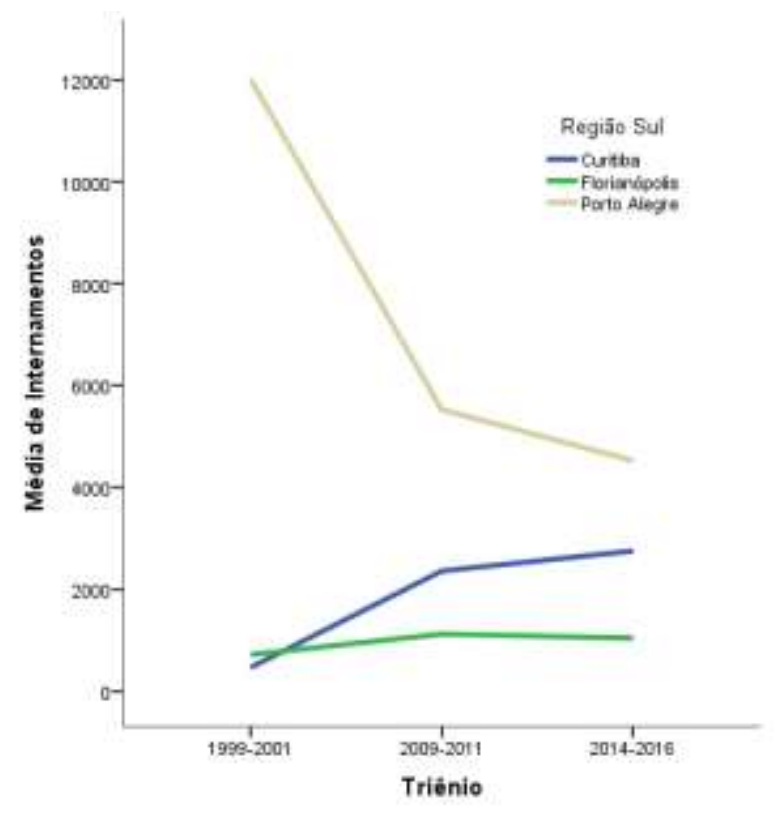

Fonte: Autores.

Observa-se que na região Centro-Oeste houve crescimento maior ao longo dos anos para Cuiabá (4218.0) e Brasília (2624.0), e decréscimo para Campo Grande (459.0) e Goiânia (720.3).

Já na região Nordeste, há inúmeras variações. Entretanto, destaca-se o crescimento para Maceió (2666.7) e Recife (4421.3), sendo esse acréscimo em meados dos anos 2009 para Maceió e uma média elevada em 1999 que decresceu em 2009 e voltou a aumentar em 2014 para Recife, já em Natal (6483.3), São Luís (1119.3) e em Fortaleza (1794.3), houve diminuição da média de ocorrência.

Na região Norte, podemos observar um grande e significativo aumento para Porto Velho (26443.7), e as demais capitais mantiveram uma média abaixo de três mil, destacando Boa Vista (178.3) e Palmas (997.0) que tiveram números abaixo de mil.

Em relação à região Sudeste, houve crescimento em todas as capitais, sendo Belo Horizonte (5817.3) a que apresentou maiores ocorrências no decorrer dos anos, seguida de São Paulo (4303.3).

A região Sul apresentou pequeno acréscimo para Curitiba e decréscimo importante para Porto Alegre.

A Tabela 2 mostra os coeficientes de correlação entre o total de internamentos e o IDH de cada capital avaliado. Houve correlação positiva, forte e significativa entre o IDH e a média de internamentos no período para Belo Horizonte $(r=1.000$ e p=0,002), Boa Vista $(r=1.00$ e p=0,010), São Paulo $(r=1.00$ e p=0,001) e Vitória $(r=0,999$ e p=0,021). 
Table 2. Correlation coefficients of total of internship in Brazil.

\begin{tabular}{|c|c|c|c|c|}
\hline & Mean of Internships & Mean of IDH & Correlation coefficient & p-value \\
\hline Aracaju & 1588.7 & 0,654 & -0.415 & 0.728 \\
\hline Belém & 2265.3 & 0,651 & 0.626 & 0.569 \\
\hline Belo Horizonte & 5817.3 & 0,713 & 1.000 & 0.002 \\
\hline Boa Vista & 178.3 & 0,643 & 1.000 & 0.010 \\
\hline Brasília & 2624.0 & 0,722 & 0.972 & 0.152 \\
\hline Campo Grande & 459.0 & 0,673 & -0.500 & 0.667 \\
\hline Cuiabá & 4218.0 & 0,682 & 0.825 & 0.383 \\
\hline Curitiba & 1857.0 & 0,738 & 0.970 & 0.157 \\
\hline Florianópolis & 958.3 & 0,765 & 0.767 & 0.444 \\
\hline Fortaleza & 1794.3 & 0,651 & -0.818 & 0.390 \\
\hline Goiânia & 720.3 & 0,705 & -0.500 & 0.667 \\
\hline João Pessoa & 4698.3 & 0,653 & -0.840 & 0.365 \\
\hline Macapá & 1011.7 & 0,627 & -0.514 & 0.656 \\
\hline Maceió & 2666.7 & 0,604 & 0.500 & 0.667 \\
\hline Manaus & 2694.3 & 0,620 & 0.797 & 0.413 \\
\hline Natal & 6483.3 & 0,666 & -0.622 & 0.573 \\
\hline Palmas & 997.0 & 0,627 & -0.270 & 0.826 \\
\hline Porto Alegre & 7355.0 & 0,736 & -0.953 & 0.157 \\
\hline Porto Velho & 26443.7 & 0,615 & 0.873 & 0.325 \\
\hline Recife & 4421.3 & 0,669 & 0.285 & 0.816 \\
\hline Rio Branco & 1873.0 & 0,601 & 0.989 & 0.095 \\
\hline Rio de Janeiro & 1673.7 & 0,718 & 0.877 & 0.319 \\
\hline Salvador & 2524.0 & 0,659 & 0.500 & 0.667 \\
\hline São Luís & 1109.3 & 0,663 & 0.789 & 0.421 \\
\hline São Paulo & 4303.3 & 0,721 & 1.000 & 0.001 \\
\hline Teresina & 171.3 & 0,627 & 0.608 & 0.584 \\
\hline Vitória & 3308.7 & 0,749 & 0.999 & 0.021 \\
\hline
\end{tabular}

Fonte: Autores.

\section{Discussão}

Os resultados encontrados no presente estudo nos sugerem que, ao longo do tempo analisado, os internamentos por sepse aumentaram em determinadas regiões e decresceram na minoria. Entre as regiões que registraram aumento, destacar o Nordeste, representado com significância estatística por Maceió, a região Norte por Porto Velho e Boa vista e, por fim, no Sudeste pelo Rio de Janeiro.

As evidências científicas nos apontam inúmeras variáveis correlacionadas aos fatores de risco para a ocorrência da sepse, e que esse crescimento tem ocorrido mundialmente (Tillmann \& Wunsch, 2018), e em território nacional (Lobo et al., 2019). Um dos fatores de relevância nesse aumento de internamentos por sepse estima-se ser o crescimento considerável da população idosa que, em 10 anos, cresceu 40,3 \%, entre os anos de 2002 e 2012. Ressalta-se que em 2010, havia no país 20,5 milhões de idosos, e estima-se para 2040, mais que o dobro, representando 23,8\% da população brasileira (Miranda et al., 2016).

É notório que a fisiologia do organismo ao decorrer dos anos passa por algumas modificações e torna a população idosa mais suscetível ao desenvolvimento de doenças crônicas não transmissíveis, tais como doenças endócrinas, do aparelho respiratório, neoplasias e doenças do aparelho circulatório (Duarte et al., 2018). Por consequência, esses indivíduos passam a buscar por assistência à saúde com mais frequência, o que resulta em exposição a microrganismos nos ambientes hospitalares, 
a procedimentos complexos e invasivos, hospitalização por maior período, que podem acarretar em infecções com possíveis desfechos em sepse (Nunes et al., 2017).

A atenção primária tem um papel de importante relevância, visto que se trata de porta de entrada a usuários do Sistema Único de Saúde. E as equipes de estratégia de saúde da família são o vínculo usuário-equipes de saúde, sendo imprescindível o conhecimento da população e da região que a unidade de saúde abrange, tornando possível assim a assistência desde a promoção, prevenção e recuperação de saúde, e, por conseguinte, que diminuam os internamentos e os riscos de uma exposição a ambiente de cuidados de saúde hospitalar (Rodrigues, Alvarez \& Rauch, 2019; Schenker \& Costa, 2019).

Outro fator relevante voltado para o âmbito primário diz respeito aos pré-natais efetivos, pois a faixa etária de neonatos compõe uma das que apresentam maior incidência (Dados do Observatório Mundial da Saúde, 2016), e estudos demonstram que complicações gestacionais tais como infecções, baixa adesão e periodicidade de consultas estão associados a partos com complicações, recém-nascidos pré-termo, baixo peso, e com maior permanência no hospital em unidades de terapia intensiva, e, portanto, maior exposição a procedimentos invasivos, entre outros desfechos desfavoráveis (Liang, 2018; Procianoy \& Silveira, 2020; Lôbo et al., 2019).

O estudo SPREAD (Sepsis PREvalence Assessment Database), realizado no Brasil, demonstrou que referente à estrutura e aos recursos de saúde, 26,8\% dos hospitais não dispõem de laboratórios adequados para obtenção de exames, tais como lactato e 10,5\% não tinham acesso a meios de identificação de microrganismo em tempo oportuno para implementação de tratamento efetivo, sendo o tratamento ainda afetado pela falta de antibióticos de amplo espectro em 10,5\% das UTIs, o que impacta negativamente no quadro dos pacientes sépticos (Taniguchi, 2019).

Contudo, dando continuidade ao que se refere à falta de recursos para a assistência, frisamos que essa variável reflete em resultados desfavoráveis, pois é fato que em ambientes que possuem mais recursos para diagnóstico, este acontece precocemente e resulta em um melhor prognóstico (Rhodes et al., 2015).

Ainda ao diagnóstico correto em tempo oportuno, o mesmo assegura o tratamento eficaz e adequado. Segundo Seymour e colaboradores (Seymour et al., 2017), quanto mais prontamente forem implementados os pacotes de abordagem frente ao paciente séptico, resultados melhores serão alcançados se comparados a abordagens inoportunas. Isto posto, faz-se necessária a incumbência de autoridades acerca de viabilizar recursos para uma assistência apropriada e efetiva.

Um estudo realizado na Califórnia, nos EUA, demonstrou melhores resultados frente à adesão de políticas direcionadas à sepse, entre elas destacamos a padronização de critérios de diagnóstico; entre os resultados foi possível demonstrar que, com a adoção dessas políticas, foi possível fornecer diagnóstico mais preciso, e, assim, diminuir a mortalidade (Gohil et al., 2016).

Além desses aspectos acima mencionados, temos outro importante fator negativo, que diz respeito ao treinamento e despreparo de equipes de saúde referentes à atualização contínua e à qualificação para implementação dos bundles, desenvolvido pela Campanha: Sobrevivendo à Sepse. Segundo o mapa regional brasileiro, publicado no relatório de atividades de 2018 do ILAS, os hospitais das capitais do Nordeste, entre 5, 6 e 10 hospitais de cada estado aderiram à capacitação, em contrapartida, nas capitais das regiões Sudeste e Sul entre 11, 20 e mais de 20 hospitais de cada estado participaram dessa capacitação e atualização, dessa maneira, são mais instituições atualizadas e capacitadas, o que reflete nos resultados obtidos no estudo em questão (ILAS, 2018).

A questão sociodemográfica e de condições e estilo de vida da população, tal qual ocorre em outras patologias, pode corroborar com os agravos em saúde e na necessidade de internamentos com desfecho em sepse.

No presente estudo, apresentamos as capitais em que ocorreram uma taxa significativa e elevada dos internamentos e o IDH dessas capitais se mostrou superior, se comparado com as demais, sendo elas: Belo Horizonte, São Paulo e Vitória. 
Sugere-se que quanto mais o IDH estiver adequado e em progresso, o país investirá em políticas de saúde pública voltadas à obtenção da qualidade de assistência à saúde, o que reflete em inúmeras variáveis favoráveis.

Portanto, o crescimento de internamentos não está intimamente relacionado com cenário desfavorável, pois a região Sudeste, todas as capitais apresentaram crescimento e a correlação com o IDH se apresentou de modo significativo, algumas capitais tais como: Belo Horizonte, tem uma média considerável (5817.3) de crescimento no período histórico estudado e correlacionado $(0,002)$ a um valor razoável de IDH $(0,713)$ o que sugere-se que a mesma dispõe de mais recursos de infraestrutura para o oferecimento de cuidados hospitalares e reconhecimento da sepse.

Já na região Sul destacamos Curitiba que manteve uma linha com poucas oscilações, o que sugere que por tratar-se de uma capital que é considerada economicamente como influência global, está entre as capitais que possuem os melhores índices de educação e PIB, e consequentemente infraestrutura mais adequada se comparada as demais, recursos de saúde que possibilitam mais assistência à saúde, o que explica-se pela linha sem significativos crescimentos ou decréscimos (Instituto Brasileiro de Geografia e Estatística. [IBGE], 2020).

Além disso citamos o fato que a saúde no Brasil perpassou por uma linha do tempo de conquistas desde 1990 e no que tange a essa linha histórica, houve a criação e o desenvolvimento de um sistema de saúde com princípios que antes não existiam, tais como: universalidade, integralidade e igualdade que surgiram a partir da constituição de 1988 (Santos, 2018), sem esmiuçar detalhes, porém não abstendo-se de mencionar, o modelo de atenção à saúde que também teve mudanças significativas, ou seja, de excludente para sanitarista e viabilizaram importantes resultados ao longo dos anos seguintes, surgindo assim maior número de indivíduos com acesso a assistência à saúde passando pela promoção, prevenção e recuperação de saúde (Teixeira et al., 2018).

No entanto, há muito o que intervir no cenário atual sobre sepse, pois a literatura nos demonstra altas taxas de ocorrência e mortalidade em países desenvolvidos citados anteriormente, Alemanha e Espanha apresentam IDH de 0,916 e 0,876 respectivamente e altas taxas de ocorrência e mortalidade por sepse (Marx et al., 2016; Blanco et al., 2008). O que valida a associação IDH razoável e média relativamente alta de internamentos em conformidade com o estudo em questão. Entretanto, mesmo com taxas elevadas, esses países apresentam uma considerável vantagem se confrontado a países de baixa e média renda (Machado \& Azevedo, 2018).

Outro fator importante diz respeito ao conhecimento e adesão da população referente ao autocuidado, o qual está diretamente associado aos índices educacionais da população e ao IDH. Uma pesquisa realizada em Porto Alegre/RS demonstrou que somente $19,1 \%$ têm conhecimento sobre o que é sepse e sugeriu que o fator renda está associado ao maior conhecimento sobre sepse, sendo assim, corrobora a respeito da associação IDH alto/melhor conhecimento, e, afeta diretamente a prevenção dos fatores de risco e da sepse propriamente dita (Moretti et al., 2019).

$\mathrm{Na}$ Alemanha, um estudo realizado com uma população $>60$ anos, demonstrou que 88,6\% já tinham ouvido falar sobre o assunto, porém a minoria sabia pontos importantes tais como a imunização na prevenção da sepse, e mais uma vez a relação escolaridade e renda se mostraram pontos fortes em relação a melhores resultados (Eitze et al., 2019).

Ressalta-se ainda o contexto mais preocupante em países em desenvolvimento, se comparado aos desenvolvidos. Fleishmann e autores (2016), indicam que a realidade está além do que conhecemos e sugerem que $87 \%$ da população têm poucos dados robustos a respeito da epidemiologia da sepse.

Os resultados mostram que a sepse é importante problema de saúde coletiva, indica as regiões mais acometidas e onde é preciso intervir para o avanço na qualidade de assistência à saúde e do progresso nos resultados esperados.

No que diz respeito às limitações da pesquisa, apontamos o tipo de estudo, pois se trata de um estudo do tipo ecológico, com dados secundários e uso de bases de dados com as limitações deste tipo de abordagem. 
Em contraposição enfatizamos que remete a um estudo incomum no Brasil, estudo esse que agrega valor pois demonstra indicadores ajustados para as causas mal definidas. Logo assegura a exclusão de considerável parcela dos efeitos das subnotificações nas regiões analisadas entre as capitais. Também ressaltamos ainda que detalha o método e instrui. Portanto, há a possibilidade de ser replicado por outros pesquisadores.

\section{Conclusão}

Podemos concluir que os internamentos por sepse tiveram crescimento significativo em Boa Vista, Maceió, Porto Velho e Rio de Janeiro e redução para Campo Grande e Goiânia nos períodos avaliados. As capitais onde houve correlação entre o IDH e o número médio de internações no triênio foram Belo Horizonte, Boa Vista, São Paulo e Vitória. Sendo assim, torna viável o conhecimento das regiões com mais fragilidades e onde se faz crucial a implementação pontual de políticas públicas de saúde com a finalidade de progresso no cenário atual. Nosso estudo sugere necessidade de pesquisas mais precisas para conhecimento da morbimortalidade por sepse, em países de baixa e média renda, a fim de tornar possível implementações de intervenções direcionadas para melhores resultados.

\section{Agradecimentos}

Agradecemos à Universidade Estadual de Ponta Grossa, Fundação Municipal de Saúde da Prefeitura Municipal de Ponta Grossa e AAC\&T Consultoria em Pesquisa LTDA.

\section{Referências}

Alves, J. B., Gabania, F. L., Ferraria, R. A. P., Taclaa, M. T. G. M., \& Júniora, A. L. (2018). Neonatal sepsis: mortality in a municipality in Southern Brazil, 2000 to 2013. Revista Paulista de Pediatria. 36(2):132-140. https://doi.org/10.1590/1984-0462/;2018;36;2;00001

Blanco, J., Muriel-Bombín, A., Sagredo, V., Taboada, F., Gandía, F., Tamayo, L., Collado, J., García-Labattut A., Carriedo, D., De Frutos, M. V. M., López, M. J., Caballero, A., Guerra, J., Álvarez, B., Mayo, A., Villar, J., \& the Grupo de Estudios y Análisis en Cuidados Intensivos (G.R.E.C.I.A.). (2008). Incidence, organ dysfunction and mortality in severe sepsis: a Spanish multicentre study. Critical Care. 12(6):R158. https://doi: 10.1186/cc7157.

Dados do Observatório Mundial da Saúde da Organização Mundial da Saúde (GHO). (2016). Causes of Child Mortality. http://www.who.int/gho/child_health/mortality/causes/en/.

Duarte, Y. A. O., Nunes, D. P., De Andrade, F. B., Corona, L. P., De Brito, T. R. P., Dos Santos, J. L. F., \& Lebrão, M. L. (2018). Fragilidade em idosos no município de São Paulo: prevalência e fatores associados. Revista Brasileira de Epidemiologia. 21(2). http://doi: 10.1590/1980-549720180021

Eitze, S., Fleischmann-Struzek, C., Betsch, C., \& Reinhart, K. (2018). Vaccination 60+ study group. Determinants of sepsis knowledge: a representative survey of the elderly population in Germany. Critical Care. 22(1):273. https://doi: 10.1186/s13054-018-2208-5.

Fleischmann C., Scherag A., Adhikari N. K., Hartog C. S., Tsaganos T., Schlattmann P., Angus D. C., Reinhart K., \& International Forum of Acute Care Trialists. (2016). Assessment of Global Incidence and Mortality of Hospital-treated Sepsis. Current Estimates and Limitations. American Journal of Respiratory and Critical Care Medicine. Feb 1;193(3):259-72. 10.1164/rccm.201504-0781OC.

Gohil, S. K., Cao, C., Phelan, M., Tjoa T., Rhee C., Platt R., \& Huang, S. S. (2016). Impact of policies on the rise in sepsis incidence, 2000-2010. Clinical Infectious Disease. 62(6):695-703. http://doi: 10.1093 / cid / civ1019.

Instituto Brasileiro de Geografia e Estatística. (IBGE) (2020). Cidades. https://cidades.ibge.gov.br/brasil/pr/curitiba/panorama.

Instituto Latino Americano de Sepse. (ILAS) (2018). Implementação de Protocolo Gerenciado de Sepse Protocolo Clínico. Atendimento ao paciente adulto com sepse / choque séptico. https://www.ilas.org.br/assets/arquivos/ferramentas/protocolo-de-tratamento.pdf

Instituto Latino Americano de Sepse. (ILAS). (2018). Relatório de atividades. Ano de referência 2018. https://ilas.org.br/assets/arquivos/upload/relatorios/relatorio-de-atividades-2018.pdf.

Kahlon, S., Pederson, J., Majumdar, S. R., Belga, S., Lau, D., Fradette, M., Boyko, D., Bakal, J. A., Johnston, C., Padwal, R. S., \& McAlister, F. A. (2015). Associação entre fragilidade e desfechos em 30 dias após a alta hospitalar. Canadian Medical Association Journal (CMAJ). 187 (11), $799-804$. https://doi.org/10.1503/cmaj.150100

Kim, H. I., \& Park, S. (2019). Sepsis: Early Recognition and Optimized Treatment. Tuberculosis \& Respiratory Diseases (Seoul). 82(1):6-14. https://doi: $10.4046 / \operatorname{trd} .2018 .0041$.

Lam, S. M., Lau, A. C., Lam., R. P., \& Yan, WW. (2017). Clinical management of sepsis. Hong Kong Medical Journal. 23(3):296-305. $10.12809 / \mathrm{hkmj} 165057$. 
Levy, M. M., Rhodes, A., Phillips, G. S., Townsend, S. R., Schorr C. A., Beale R., Osborn, T., Lemeshow, S., Chiche, J-D., Artigas, A., \& Dellinger R. P. (2015). Surviving Sepsis Campaign: association between performance metrics and outcomes in a 7.5-year study. Critical Care Medicine. 43(1):3-12. 10.1097/CCM.0000000000000723.

Liang, L. D., Kotadia, N., English, L., Kissoon, N., Ansermino, J. M., Kabakyenga, J., Lavoie, P. M., \& Wiens, M. O. (2018). Predictors of Mortality in Neonates and Infants Hospitalized with Sepsis or Serious Infections in Developing Countries: A Systematic Review. Frontics in Pediatrics. 4:6:277. http://doi: 10.3389/fped.2018.00277

Lôbo, I. K. V., Konstantyner, T., Areco, K. C. N., Vianna, R. P. T., \& Taddei, J. A. A. C. (2019). Internações por Condições Sensíveis à Atenção Primária de Menores de um ano, de 2008 a 2014, no estado de São Paulo, Brasil. Ciência \& saúde coletiva. pp.24 p.9. http://dx.doi.org/10.1590/141381232018249.29932017 .

Lobo, M. S., Rezende, E., Mendes, C. L., \& Oliveira, M. C. (2019). Mortalidade por sepse no Brasil em um cenário real: projeto UTIs Brasileiras. Revista Brasileira Terapia Intensiva. https://doi.org/10.5935/0103-507x.20190008.

Machado, F. R., \& Azevedo, L. C. P. (2018). Sepsis: A Threat That Needs a Global Solution. Critical Care Medicine. 46(3):454-459. 10.1097/CCM.0000000000002899.

Machado, F. R., Cavalcanti, A. B., Bozza, F. A., Ferreira, E. M., Carrara, F. S. A., Souza, J. L., Caixeta, N., Salomão, R., Angus, D. C., Azevedo, L. C. P., \& SPREAD Investigators; Latin American Sepsis Institute Network. (2017). The epidemiology of sepsis in Brazilian intensive care units (the Sepsis PREvalence Assessment Database, SPREAD): an observational study. The Lancet Infectious Disease. 17(11):1180-9. https://doi.org/10.1016/S1473-3099(17)30322-5.

SepNet Critical Care Trials Group. (2016). Incidence of severe sepsis and septic shock in German intensive care units: the prospective, multicentre INSEP study. Intensive Care Medicine 42. 1980-1989. https://doi.org/10.1007/s00134-016-4504-3

Miranda, G. M. D., Mendes, A. C. G., \& Silva, A. L. A. (2016). Population aging in Brazil: current and future social challenges and consequences. Revista Brasileira de Geriatria e Gerontolongia. 19(3), 507-519. http://dx.doi.org/10.1590/1809-98232016019.150140.

Moretti, M. M. S., Urbanetto, J. S., Nascimento, A. P., Rodrigues, A. G., Silva, D. R., Ramos, T., \& Rockenbach, V. (2019). Sepse e IAM: conhecimento da população frequentadora de parques e acompanhantes de pacientes. Revista Gaúcha de Enfermagem. pp.40. https://doi.org/10.1590/19831447.2019.20180299.

Muller, E. V., \& Gimeno, S. G. (2015). Mortalidade por doenças cardiovasculares segundo gênero e idade no Estado do Paraná: 1979 a 1981 e 2006 a 2008. Caderno de Saúde Coletiva. pp.23 no.1. http://dx.doi.org/10.1590/1414-462X201500010003.

Nunes, B. P., Soares, M. U., Wachs, L. S., Volz, P. M., Saes, M. O., Duro, S. M. S., Thumé, E., \& Facchini, L. A. (2017). Hospitalization in older adults: association with multimorbidity, primary health care and private health plan. Revista de Saúde Pública. 4;51:43. http://doi: 10.1590/S15188787.2017051006646 .

Pereira, A. S., Shitsuka, D. M., Parreira, F. J., \& Shitsuka, R. (2018). Metodologia da pesquisa científica. UFSM. https://repositorio.ufsm.br/bitstream/handle/1/15824/Lic_Computacao_Metodologia-Pesquisa-Cientifica.pdf?sequence=1 .

Procianoy, R. S., \& Silveira, R. C. (2020). The challenges of neonatal sepsis management. Jornal de Pediatria. 96 (S1):80-86. https://doi.org/10.1016/j.jped.2019.10.004.

Programa das Nações Unidas para o Desenvolvimento (PNUD) (2013). Atlas do Desenvolvimento Humano no Brasil. http://www.br.undp.org/content/brazil/pt/home/idh0/rankings/idhm-municipios-2010.html.

Programa das Nações Unidas para o Desenvolvimento (PNUD), Instituto de Pesquisa Econômica Aplicada (IPEA)., \& Fundação João Pinheiro (FJP). O Índice de Desenvolvimento Humano Municipal Brasileiro, (2016). https://www.br.undp.org/content/brazil/pt/home/library/idh/Atlas/o-idh-brasileiro0.html.

Rhodes, A., Phillips, G., Beale, R., Cecconi, M., Chiche, J. D., De Backer D., Divatia, J., Du, B., Evans, L., Ferrer, R., Girardis, M., Koulenti, D., Machado, F., Simpson, S. Q., Tan, C.C., Wittebole, X., \& Levy, M. (2015). The Surviving Sepsis Campaign bundles and outcome: results from the International Multicentre Prevalence Study on Sepsis (the IMPreSS study). Intensive Care Medicine. 41(9):1620-8. http://doi: 10.1007 / s00134-015-3906-y.

Rodrigues, M. M., Alvarez, A. M., \& Rauch, K. C. (2019). Tendência das internações e da mortalidade de idosos por condições sensíveis à atenção primária. Revista Brasileira de Epidemiologia. 22, e190010. http://dx.doi.org/10.1590/1980-549720190010.

Rudd, K. E., Johnson, S. C., Agesa, K. M., Shackelford, K. A., Tsoi, D., Kievlan, D. R., Rudd, K., Johnson, S. C., Agesa, K. M., Shackelford, K. A., Tsoi, D., Kievlan, D. R., Colombara, D. V., Ikuta, K. S., Kissoon, N., Finfer, S., Fleischmann-Struzek, C., Machado, F. R., Reinhart, K. K., \& Naghavi, M. (2020). Global, regional, and national sepsis incidence and mortality, 1990-2017: analysis for the Global Burden of Disease Study. The Lancet. 395(10219):200-211. https://doi: 10.1016/S0140-6736(19)32989-7.

Santos, N. R. D. (2018). 30 years of SUS: the beginning, the pathway and the target. Ciência \& Saúde Coletiva. 23(6):1729-1736. Portuguese, English. doi: $10.1590 / 1413-81232018236.06092018$.

Schenker, M., \& Costa, D. H. (2019). Avanços e desafios da atenção à saúde da população idosa com doenças crônicas na Atenção Primária à Saúde. Ciência \& Saúde Coletiva. 24(4),1369-1380. http://doi: org/10.1590/1413-81232018244.01222019>.

Seymour, C. W., Gesten. F., Prescott, H. C., Friedrich, M. E., Iwashyna, T. J., Phillips, G. S., Lemeshow, S., Osborn, T., Terry, K. M., \& Levy, MM. (2017). Time to treatment and mortality during mandated emergency care for sepsis. The New England Journal of Medicine. 376(23):2235-44. http://doi: 10.1056 / NEJMoa1703058 
Research, Society and Development, v. 10, n. 5, e31610514977, 2021

(CC BY 4.0) | ISSN 2525-3409 | DOI: http://dx.doi.org/10.33448/rsd-v10i5.14977

Singer, M., Deutschman, C. S., Seymour, C. W., Shankar-Hari, M., Annane, D., Bauer, M., . . .Bellomo, R., Bernard, G. R., Chiche, J-D., Coopersmith, C. M., Hotchkiss, R. S., Levy, M. M., Marshall, J. C., Martin, G. S., Opal, S. M., Rubenfeld, G. D., Van der Poll, T., Vincent, J-L., \& Angus, D. C. (2016). The Third International Consensus Definitions for Sepsis and Septic Shock (Sepsis-3). Clinical Review \& Education. 23:315. https://doi: 10.1001/jama.2016.0287.

Taniguchi, L. U., Azevedo, L. C. P., Bozza, F. A., Cavalcanti, A. B., Ferreira, E. M., Carrara, F. S. A. C., Sousa, J. L., Salomão, R., \& Machado, F. R. (2019). Disponibilidade de recursos para tratamento da sepse no Brasil: uma amostra aleatória de instituições brasileiras. Revista Brasileira de Terapia Intensiva. pp.31 no.2. http://doi.org/10.5935/0103-507x.20190033.

Teixeira, M. G., Costa, M. D. C. N., Carmo, E. H., Oliveira, W. K., \& Penna, G. O. (2018). Health surveillance at the SUS: development, effects and perspectives. Ciência \& Saúde Coletiva. 23(6):1811-1818. 10.1590/1413-81232018236.09032018.

Tillmann, B., \& Wunsch, H. (2018). Epidemiology and Outcomes. Critical Care Clinics. 34(1):15-27. https://doi: 10.1016/j.ccc.2017.08.001.

Westphal, G. A., \& Lino, A. S. (2015). Rastreamento sistemático é a base do diagnóstico precoce da sepse grave e choque séptico. Revista Brasileira de Terapia Intensiva. 27(2):96-101. 2015. https://doi:10.5935/0103-507X.20150018. 